\title{
Converse Lyapunov theorems for infinite-dimensional nonlinear switching systems
}

\author{
Ihab Haidar, Yacine Chitour, Paolo Mason and Mario Sigalotti
}

\begin{abstract}
In this paper, we provide two converse Lyapunov theorems in the framework of nonlinear infinitedimensional switching systems. Our results characterize uniform exponential stability with respect to the switching law through the existence of both coercive and non-coercive Lyapunov functionals. The starting point for our arguments is a generalization of the well-known Datko lemma to the case of nonlinear infinite-dimensional switching systems.
\end{abstract}

Index Terms-infinite-dimensional systems, nonlinear systems, switching systems, converse Lyapunov theorems.

\section{INTRODUCTION}

To deal with stability issues of switching systems many approaches based on Lyapunov techniques have been developed. In particular many sufficient conditions for stability are based on the existence of multiple Lyapunov functions (see e.g. [1]) or common Lyapunov functions (see e.g. [3]). For linear finite-dimensional switching systems, it is well known that the existence of a common Lyapunov function is a necessary and sufficient condition for their uniform asymptotic stability [3]. The extension of such a result to infinite-dimensional linear systems has been done in [7]. To the best of our knowledge, the uniform exponential stability of infinitedimensional nonlinear switching systems has not yet been developed in the literature.

Various works have been recently devoted to the characterization of the stability of infinite-dimensional systems in Banach space $X$ through non-coercive Lyapunov functionals (see, e.g., [5], [7], [10], [11] and [12]). By non-coercive Lyapunov functional, we simply mean a positive definite functional decaying along the trajectories of the system which satisfies

$$
0<V(x) \leq \alpha(\|x\|), \quad \forall x \in X \backslash\{0\},
$$

Ihab Haidar is with Laboratoire Quartz EA 7393, ENSEA, CergyPontoise, France ihab.haidar@ensea.fr

Yacine Chitour and Paolo Mason are with Laboratoire des Signaux et Systèmes (L2S), CNRS, CentraleSupélec, Université Paris-Sud, Université Paris-Saclay, Gif-sur-Yvette, France \{yacine. chitour, paolo.mason\}el2s. centralesupelec.fr

Mario Sigalotti is with Laboratoire Jacques-Louis Lions, Inria, Sorbonne Université, Université Paris-Diderot, CNRS, Paris, France mario.sigalotti@inria.fr where $\alpha \in \mathcal{K}_{\infty}$, the class of continuous increasing bijections from $\mathbb{R}_{+}$to $\mathbb{R}_{+}$. Such a function $V$ would be coercive if there would exists $\alpha_{0} \in \mathcal{K}_{\infty}$ such that $V(x) \geq \alpha_{0}(\|x\|)$, for $x \in X$. In the literature, Lyapunov functionals satisfying (1) are equivalently called weaklydegenerate (see, e.g., [5]). Recall, from [2], that the exponential stability of a linear $C_{0}$-semigroup $(T(t))_{t \geq 0}$ on a complex Hilbert space $H$ is equivalent to the existence of a positive Hermitian endomorphism $B$ on $H$ such that the relation $2 \Re(B A x, x)=-\|x\|^{2}$ holds for every $x$ in the domain of $A$, the infinitesimal generator of the semigroup $(T(t))_{t \geq 0}$. In this case, we have

$$
(B x, x)=\int_{0}^{+\infty}\|T(t) x\|^{2} d t, \quad \forall x \in H,
$$

and, as reported in [13], the functional $V: X \rightarrow$ $\mathbb{R}^{+}$defined by $V(x)=(B x, x)$ is in general a noncoercive Lyapunov functional, i.e., $\sqrt{V}$ is not necessarily equivalent to a norm on $H$ since there does not exist in general a positive real number $c$ such that

$$
\int_{0}^{+\infty}\|T(t) x\|^{2} d t \geq c\|x\|^{2}, \quad \forall x \in H .
$$

Of course, in the case of finite-dimensional spaces, an exponential stable linear semigroup $(T(t))_{t \geq 0}$ is given by $\left(e^{t A}\right)_{t \geq 0}$ with $A$ Hurwitz and hence inequality (3) holds true. A complete characterization of $C_{0^{-}}$ semigroups defining a norm on $H$ is given in [13].

Another interesting point resulting from [2] is the well-known Datko lemma which characterizes the asymptotic behavior of a linear $C_{0}$-semigroup $(T(t))_{t \geq 0}$ on a Hilbert space $H$ through the convergence of the integral term given by equation (2). More precisely, the linear $C_{0}$-semigroup $(T(t))_{t \geq 0}$ is globally exponentially stable if and only if, for every $x \in H$, the integral in (2) is convergent. Datko's lemma still holds true in a general Banach space, as proved in [13]. This result is extended in [8] to the framework of nonlinear semigroups with the only difference that the nonlinear semigroup needs to satisfy the following boundedness property

$$
\|T(t) x\| \leq g(t)\|x\|, \quad \forall t \geq 0, \forall x \in X,
$$


for some positive and continuous function $g$ (a condition which is automatically satisfied for linear $C_{0^{-}}$ semigroups [14]).

In this work we generalize Datko's lemma to the case of infinite dimensional nonlinear switching systems in Banach space, combining the approaches of [7], [8], and [16]. The paper [8] provides a nonlinear version of Datko's lemma, while [7] and [16] deal with linear switching systems. Thanks to such a generalization, we prove that the existence of non-coercive and coercive Lyapunov functionals is necessary and sufficient for the uniform global exponential stability. The result dealing with a non-coercive Lyapunov functional is better suited to derive a stability result, while the result dealing with a coercive Lyapunov functional provides more information on a nonlinear switching system that is known to be globally uniformly exponentially stable, by tightening the properties satisfied by the Lyapunov functional. The main difference with respect to the characterization provided in [12] is that we focus here on necessary and sufficient conditions for exponential stability, rather than asymptotic stability. Converse Lyapunov theorems are helpful tools in the analysis of interconnected systems (such as in [6]) and input-to-state stability (see, e.g., [9] and [15]), for example.

The paper is organized as follows. Section II presents the list of notations and definitions in use. In Section III, we extend Datko's lemma to nonlinear switching systems. A first converse theorem with non-coercive Lyapunov functional is given in Section IV and a second converse theorem with coercive Lyapunov functional is given in Section V.

\section{NOTATIONS AND DEFINITIONS}

By $(X,\|\cdot\|)$, we denote a Banach space with norm $\|\cdot\|$ and by $B_{X}(0, r)$ the closed ball of center 0 and radius $r \geq 0$. We use $\mathbb{R}_{+}$and $\mathbb{R}_{+}^{*}$ to denote the set of non-negative real numbers and positive real numbers respectively and we set $\overline{\mathbb{R}}=\mathbb{R} \cup\{ \pm \infty\}$. Let $\mathcal{Q}$ be a nonempty index set. We denote by $\mathcal{S}$ the set of piecewise constant switching signals $\sigma: \mathbb{R}_{+} \rightarrow \mathcal{Q}$. For all $\sigma \in \mathcal{S}$ and all $\tau \geq 0$, the $\tau$-shifted signal $\sigma_{\tau} \in \mathcal{S}$ is defined as $\sigma_{\tau}(s)=\sigma(\tau+s)$.

We next give the definition of a strongly continuous nonlinear semigroup on a Banach space $X$.

Definition 1: Let $T(t): X \rightarrow X, t \geq 0$, be a family of nonlinear maps. We say that $(T(t))_{t \geq 0}$ is a strongly continuous nonlinear semigroup if the following holds ${ }^{1}$

i) $\forall x \in X, T(0) x=x$;

${ }^{1}$ Following the classical notation in nonlinear semigroup theory we use $T(t) x$ to denote the evaluation of the map $T(t)$ at $x$. ii) $\forall t_{1}, t_{2} \geq 0, T\left(t_{1}\right) T\left(t_{2}\right) x=T\left(t_{1}+t_{2}\right) x$;

iii) for each $x \in X$ the map $t \mapsto T(t) x$ is continuous.

Let $\sigma \in \mathcal{S}$. More precisely, assume that $\sigma \equiv \sigma_{k}$ on $\left[t_{k}, t_{k+1}\right)$, where $\left(t_{k}\right)_{k \geq 0}$ is an increasing sequence with $t_{0}=0$ and $\sigma_{k} \in \mathcal{Q}$ for $k \geq 0$. With each $\sigma_{k}$ we associate the strongly continuous nonlinear semigroup $\left(T_{\sigma_{k}}(t)\right)_{t \geq 0}$. By concatenating the flows $\left(T_{\sigma_{k}}(t)\right)_{t \geq 0}$, one can associate with $\sigma$ the nonlinear evolution operator

$$
T_{\sigma(\cdot)}(t):=T_{\sigma_{k}}\left(t-t_{k}\right) T_{\sigma_{k-1}}\left(t_{k}-t_{k-1}\right) \cdots T_{\sigma_{0}}\left(t_{1}\right),
$$

for all $t \in\left[t_{k}, t_{k+1}\right)$. The evolution operator $\left(T_{\sigma(\cdot)}(t)\right)_{t>0}$ just introduced identifies the switching nonlinear system in $X$

$$
\left\{\begin{array}{l}
x(t)=T_{\sigma(\cdot)}(t) x_{0} \\
x_{0} \in X
\end{array}\right.
$$

With the previous notations, we have the following concatenation property

$$
T_{\sigma(\cdot)}(t) x=T_{\sigma_{\tau}(\cdot)}(t-\tau) T_{\sigma(\cdot)}(\tau) x,
$$

for all $\sigma \in \mathcal{S}, 0 \leq \tau \leq t$ and $x \in X$.

The notion of uniform Lipschitz continuity of the flow with respect to initial conditions is given by the following definition.

Definition 2: We say that the flow of $\Sigma$ is uniformly (with respect to $(t, \sigma)$ ) Lipschitz on bounded subsets of $X$, if for any $r>0$ there exists $L_{r}>0$ such that $\forall x, y \in B_{X}(0, r), \forall t \geq 0, \forall \sigma \in \mathcal{S}$, we have

$$
\left\|T_{\sigma(\cdot)}(t) x-T_{\sigma(\cdot)}(t) y\right\| \leq L_{r}\|x-y\| .
$$

The notion of uniform (with respect to the switching signals) global exponential stability is given by the following definition.

Definition 3: We say that the system $\Sigma$ is 0-UGES if there exist $M, \lambda>0$ such that

$$
\left\|T_{\sigma(\cdot)}(t) x\right\| \leq M e^{-\lambda t}\|x\|, \quad \forall t \geq 0, \forall x \in X, \forall \sigma \in \mathcal{S} .
$$

We also need the following definition of the Dini derivatives of a continuous functional $V: X \rightarrow \mathbb{R}_{+}$.

Definition 4: Consider the system $\Sigma$. For a continuous functional $V: X \rightarrow \mathbb{R}_{+}$, its upper and lower Dini derivatives, denoted by $\bar{D}_{q} V: X \rightarrow \overline{\mathbb{R}}$ and $\underline{D}_{q} V: X \rightarrow \overline{\mathbb{R}}$ respectively, are defined for $x \in X$ and $q \in \mathcal{Q}$, by

$$
\bar{D}_{q} V(x)=\limsup _{h \rightarrow 0^{+}} \frac{1}{h}\left(V\left(T_{q}(h) x\right)-V(x)\right),
$$

and

$$
\underline{D}_{q} V(x)=\liminf _{h \rightarrow 0^{+}} \frac{1}{h}\left(V\left(T_{q}(h) x\right)-V(x)\right) .
$$




\section{DATKO'S TYPE THEOREM FOR NONLINEAR SWITCHING SYSTEMS}

In this section, we extend Datko's lemma [2], to the nonlinear switching infinite-dimensional system $\Sigma$.

Theorem 5: Consider the switching system $\Sigma$. Let $g: \mathbb{R}_{+} \rightarrow \mathbb{R}_{+}^{\star}$ be a continuous function, bounded from below by a positive constant, such that

$$
\left\|T_{\sigma(\cdot)}(t) x\right\| \leq g(t)\|x\|, \forall t \geq 0, \forall x \in X, \forall \sigma \in \mathcal{S} .
$$

The following statements are equivalent:

i) The system $\Sigma$ is 0-UGES,

ii) for every $p>0$, there exists $k>0$ such that, for all $x \in X$ and all $\sigma \in \mathcal{S}$, we have

$$
\int_{0}^{+\infty}\left\|T_{\sigma(\cdot)}(t) x\right\|^{p} d t \leq k^{p}\|x\|^{p},
$$

iii) there exist $p, k>0$ such that, for all $x \in X$ and all $\sigma \in \mathcal{S}$ we have

$$
\int_{0}^{+\infty}\left\|T_{\sigma(\cdot)}(t) x\right\|^{p} d t \leq k^{p}\|x\|^{p}
$$

Proof: By using Definition 3, one deduces that i) implies ii) with $k^{p}=\frac{M^{p}}{\lambda p}$. Moreover, ii) clearly implies iii). It then remains to prove that iii) implies i), which is proved in three steps. We first show that there exists $C>0$ such that

$$
\left\|T_{\sigma(\cdot)}(t) x\right\| \leq C\|x\|, \quad \forall t \geq 0, \forall x \in X, \forall \sigma \in \mathcal{S} .
$$

We then prove that, for each $0<c<1$, there exists a $t_{0}=t_{0}(c)$ such that

$$
\left\|T_{\sigma(\cdot)}(t) x\right\| \leq c\|x\|, \quad \forall t \geq t_{0}, \forall x \in X, \forall \sigma \in \mathcal{S} .
$$

The concatenation property (5) together with the previous inequality conclude the proof.

Let $(x, \sigma)$ in $X \times \mathcal{S}$. For each $t \geq 0$, it follows, from Definition 1 and inequalities (7) and (8), that

$$
\begin{aligned}
& \left\|T_{\sigma(\cdot)}(t) x\right\|^{p} \int_{0}^{t} \frac{1}{g^{p}(\tau)} d \tau \\
= & \int_{0}^{t}\left\|T_{\sigma(\cdot)}(t) x\right\|^{p} \frac{1}{g^{p}(t-\tau)} d \tau \\
= & \int_{0}^{t}\left\|T_{\sigma_{\tau}(\cdot)}(t-\tau) T_{\sigma(\cdot)}(\tau) x\right\|^{p} \frac{1}{g^{p}(t-\tau)} d \tau \\
\leq & \int_{0}^{t}\left\|T_{\sigma(\cdot)}(\tau) x\right\|^{p} d \tau \leq k^{p}\|x\|^{p} .
\end{aligned}
$$

Define $k_{1}$ by

$$
k_{1}^{p}=\int_{0}^{1} \frac{1}{g^{p}(\tau)} d \tau>0 .
$$

We observe that, for any $t \geq 1$,

$$
k_{1}^{p} \leq \int_{0}^{t} \frac{1}{g^{p}(\tau)} d \tau .
$$

We deduce from (9) and (10) that

$$
\left\|T_{\sigma(\cdot)}(t) x\right\| \leq \frac{k}{k_{1}}\|x\|, \quad \forall t \geq 1 .
$$

Combining with (7) it follows that

$$
\left\|T_{\sigma(\cdot)}(t) x\right\| \leq C\|x\|, \quad \forall t \geq 0, \forall x \in X, \forall \sigma \in \mathcal{S},
$$

where

$$
C=\max \left\{\sup _{t \in[0,1]} g(t), \frac{k}{k_{1}}\right\} .
$$

Now, let $t>0$ and $(x, \sigma)$ be fixed in $X \times \mathcal{S}$. We have,

$$
\begin{aligned}
& t\left\|T_{\sigma(\cdot)}(t) x\right\|^{p}=\int_{0}^{t}\left\|T_{\sigma(\cdot)}(t) x\right\|^{p} d \tau \\
& =\int_{0}^{t}\left\|T_{\sigma_{\tau}(\cdot)}(t-\tau) T_{\sigma(\cdot)}(\tau) x\right\|^{p} d \tau \\
& \leq C^{p} \int_{0}^{t}\left\|T_{\sigma(\cdot)}(\tau) x\right\|^{p} d \tau \leq(k C)^{p}\|x\|^{p} .
\end{aligned}
$$

It follows that

$$
\left\|T_{\sigma(\cdot)}(t) x\right\| \leq \frac{k C}{t^{1 / p}}\|x\| .
$$

So, for each $0<c<1$, there exists $t_{0}=t_{0}(c)>0$ such that

$\left\|T_{\sigma(\cdot)}(t) x\right\| \leq c\|x\|, \quad \forall t \geq t_{0}, \forall x \in X, \forall \sigma \in \mathcal{S}$.

Now, let $t \geq 0$ be fixed. There exists an integer $n \geq 0$ such that $t=n t_{0}+s$, with $0 \leq s<t_{0}$. We have

$$
\begin{aligned}
\left\|T_{\sigma(\cdot)}(t) x\right\| & =\left\|T_{\sigma_{n t_{0}}(\cdot)}(s) T_{\sigma(\cdot)}\left(n t_{0}\right) x\right\| \\
& \leq C\left\|T_{\sigma(\cdot)}\left(n t_{0}\right) x\right\| \leq C c^{n}\|x\| \\
& \leq M e^{-\lambda t}\|x\|,
\end{aligned}
$$

with $M=\frac{C}{c}$ and $\lambda=-\frac{1}{t_{0}} \ln (c)>0$.

Remark 6: Without loss of generality one may replace $g(t)$ in (7) by an exponential function. Indeed, by the concatenation property (5), it is easy to see that (7) implies

$$
\left\|T_{\sigma(\cdot)}(t) x\right\| \leq M e^{\lambda t}\|x\|, \forall t \geq 0, \forall x \in X, \forall \sigma \in \mathcal{S},
$$

with $M=\max _{s \in[0,1]} g(s)$ and $\lambda=\max \{0, \log (g(1))\}$. Notice that inequality (13) is a strong requirement on the switching system $\mathcal{S}$. Even in the linear case, and even if each mode satisfies (13), uniformly with respect to the mode, it does not follow that a similar exponential bound holds for the corresponding switching system, cf. [7, Example 1]. 


\section{FIRST CONVERSE THEOREM}

In this section, we give our main result which states that the origin of an infinite-dimensional nonlinear switching system on a Banach space is uniformly globally exponentially stable if and only if it admits a noncoercive Lyapunov function.

Theorem 7: Consider the nonlinear switching system $\Sigma$, with a flow which is uniformly Lipschitz on bounded subsets of $X$. The system $\Sigma$ is 0-UGES if and only if the following conditions hold:

i) there exists a continuous function $g: \mathbb{R}_{+} \rightarrow \mathbb{R}_{+}^{\star}$, bounded from below by a positive constant, such that for all $t \geq 0$ and for all $x \in X$,

$$
\left\|T_{\sigma(\cdot)}(t) x\right\| \leq g(t)\|x\|, \quad \forall \sigma \in \mathcal{S}
$$

ii) there exist a functional $V: X \rightarrow \mathbb{R}_{+}$, Lipschitz on bounded subsets of $X$, and $p, c>0$ such that

$$
V(x) \leq c\|x\|^{p}, \quad \forall x \in X
$$

and

$$
\underline{D}_{q} V(x) \leq-\|x\|^{p}, \quad \forall x \in X, \forall q \in \mathcal{Q} .
$$

Proof: Assume that conditions i) and ii) hold. Let $x \in X$ and $\sigma \in \mathcal{S}$. For $t \geq 0$ small enough so that the restriction of $\sigma$ to the interval $[0, t]$ is constantly equal to $q \in \mathcal{Q}$, we have from inequality (16) that

$$
\underline{D}_{q} V\left(T_{\sigma(\cdot)}(t) x\right) \leq-\left\|T_{\sigma(\cdot)}(t) x\right\|^{p},
$$

which implies that (see [4, Theorem 9])

$$
V\left(T_{\sigma(\cdot)}(t) x\right) \leq V(x)-\int_{0}^{t}\left\|T_{\sigma(\cdot)}(\tau) x\right\|^{p} d \tau .
$$

Inequality (17), together with (15), yields

$$
\int_{0}^{t}\left\|T_{\sigma(\cdot)}(\tau) x\right\|^{p} d \tau \leq c\|x\|^{p} .
$$

Thus, for all $x \in X$ and all $\sigma \in \mathcal{S}$, we have

$$
\int_{0}^{\infty}\left\|T_{\sigma(\cdot)}(\tau) x\right\|^{p} d \tau \leq c\|x\|^{p}
$$

which implies, thanks to Theorem 5, the uniform global exponential stability of system $\Sigma$.

Conversely, assume that system $\Sigma$ is 0-UGES. Thus, (14) holds with $g(t)=M e^{-\lambda t}$, for some positive constants $M$ and $\lambda$. Define the functional $V: X \rightarrow \mathbb{R}_{+}$ by

$$
V(x)=\sup _{\sigma \in \mathcal{S}} \int_{0}^{\infty}\left\|T_{\sigma(\cdot)}(t) x\right\|^{2} d t, \quad x \in X .
$$

Thanks to the uniform exponential stability of $\Sigma$, the functional $V$ is well defined. Let us prove ii) with $p=2$. It is clear that in this case inequality (15) holds true with

$$
c=M^{2} \int_{0}^{\infty} e^{-2 \lambda t} d t=\frac{M^{2}}{2 \lambda} .
$$

Let us now prove (16). For this, let us define, for each fixed $h>0$, and each $q$ fixed in $\mathcal{Q}$ the set

$$
\mathcal{S}^{h, q}=\{\sigma \in \mathcal{S}: \sigma \equiv q \text { over }[0, h]\},
$$

i.e., the set of switching signals whose restriction to the interval $[0, h]$ is constantly equal to $q$. Hence,

$$
\begin{aligned}
& V(x)=\sup _{\sigma \in \mathcal{S}} \int_{0}^{+\infty}\left\|T_{\sigma(\cdot)}(t) x\right\|^{2} d t \\
& =\sup _{\sigma \in \mathcal{S}}\left(\int_{0}^{h}\left\|T_{\sigma(\cdot)}(t) x\right\|^{2} d t+\int_{h}^{+\infty}\left\|T_{\sigma(\cdot)}(t) x\right\|^{2} d t\right) \\
& \geq \sup _{\sigma \in \mathcal{S}^{h, q}}\left(\int_{0}^{h}\left\|T_{\sigma(\cdot)}(t) x\right\|^{2} d t+\int_{h}^{+\infty}\left\|T_{\sigma(\cdot)}(t) x\right\|^{2} d t\right) \\
& =\int_{0}^{h}\left\|T_{q}(t) x\right\|^{2} d t+\sup _{\sigma \in \mathcal{S}^{h, q}} \int_{h}^{+\infty}\left\|T_{\sigma(\cdot)}(t) x\right\|^{2} d t .
\end{aligned}
$$

Furthermore, we have

$$
\begin{aligned}
& V\left(T_{q}(h) x\right)=\sup _{\sigma \in \mathcal{S}} \int_{0}^{+\infty}\left\|T_{\sigma(\cdot)}(t) T_{q}(h) x\right\|^{2} d t \\
& =\sup _{\sigma \in \mathcal{S}} \int_{h}^{+\infty}\left\|T_{\sigma(\cdot)}(t-h) T_{q}(h) x\right\|^{2} d t \\
& =\sup _{\sigma \in \mathcal{S}^{h, q}} \int_{h}^{+\infty}\left\|T_{\sigma(\cdot)}(t) x\right\|^{2} d t .
\end{aligned}
$$

Thus, (20) and (21) yields the inequality

$$
\frac{V\left(T_{q}(h) x\right)-V(x)}{h} \leq-\frac{1}{h} \int_{0}^{h}\left\|T_{q}(t) x\right\|^{2} d t .
$$

Therefore, for each $x \in X$ and each fixed $q \in \mathcal{Q}$, it follows that

$$
\begin{aligned}
& \underline{D}_{q} V(x)=\liminf _{h \rightarrow 0^{+}} \frac{V\left(T_{q}(h) x\right)-V(x)}{h} \\
& \leq \limsup _{h \rightarrow 0^{+}} \frac{V\left(T_{q}(h) x\right)-V(x)}{h} \\
& \leq \limsup _{h \rightarrow 0^{+}} \frac{1}{h}\left(-\int_{0}^{h}\left\|T_{q}(t) x\right\|^{2} d t\right) \\
& \leq-\liminf _{h \rightarrow 0^{+}} \frac{1}{h}\left(\int_{0}^{h}\left\|T_{q}(t) x\right\|^{2} d t\right) \\
& =-\|x\|^{2}
\end{aligned}
$$

establishing (16), with $p=2$. 
Finally, we prove that the functional $V$ is Lipschitz on bounded subsets of $X$. For this, let $r$ be any positive real and let $x, y \in B_{X}(0, r)$. We have

$$
\begin{aligned}
& |V(x)-V(y)|= \\
& \left|\sup _{\sigma \in \mathcal{S}} \int_{0}^{\infty}\left\|T_{\sigma(\cdot)}(t) x\right\|^{2} d t-\sup _{\sigma \in \mathcal{S}} \int_{0}^{\infty}\left\|T_{\sigma(\cdot)}(t) y\right\|^{2} d t\right| \\
& \leq\left|\sup _{\sigma \in \mathcal{S}}\left(\int_{0}^{\infty}\left\|T_{\sigma(\cdot)}(t) x\right\|^{2} d t-\int_{0}^{\infty}\left\|T_{\sigma(\cdot)}(t) y\right\|^{2} d t\right)\right| \\
& \leq \sup _{\sigma \in \mathcal{S}}\left|\int_{0}^{\infty}\left\|T_{\sigma(\cdot)}(t) x\right\|^{2} d t-\int_{0}^{\infty}\left\|T_{\sigma(\cdot)}(t) y\right\|^{2} d t\right| \\
& \leq \sup _{\sigma \in \mathcal{S}} \int_{0}^{\infty}\left|\left\|T_{\sigma(\cdot)}(t) x\right\|^{2}-\left\|T_{\sigma(\cdot)}(t) y\right\|^{2}\right| d t .
\end{aligned}
$$

Using the 0-UGES of $\Sigma$ together with the fact that the flow is uniformly Lipschitz on bounded subsets of $X$, it follows, from (23), that

$$
\begin{aligned}
& \quad|V(x)-V(y)| \\
& \leq \sup _{\sigma \in \mathcal{S}} \int_{0}^{\infty}\left(\left\|T_{\sigma(\cdot)}(t) x\right\|+\left\|T_{\sigma(\cdot)}(t) y\right\|\right) \\
& \left.\leq 2 M r \sup _{\sigma \in \mathcal{S}} \int_{0}^{\infty} e^{-\lambda t}\left\|T_{\sigma(\cdot)}(t) x\right\|-\left\|T_{\sigma(\cdot)}(t) y\right\|\right) d t \\
& \leq 2 M r L_{r} \int_{0}^{\infty} e^{-\lambda t}\|x-y\| d t \\
& =2 \frac{M}{\lambda} r L_{r}\|x-y\| .
\end{aligned}
$$

This implies that the functional $V$ is Lipschitz on bounded subsets of $X$.

\section{SECOND CONVERSE THEOREM}

We prove in this section a second converse Lyapunov theorem stating that the origin of an infinite-dimensional nonlinear switching system on a Banach space is uniformly globally exponentially stable if and only if there exists a coercive Lyapunov function for this system.

Theorem 8: Consider the nonlinear switching system $\Sigma$, with a flow which is uniformly Lipschitz on bounded subsets of $X$. The system $\Sigma$ is 0-UGES if and only if there exists a functional $V: X \rightarrow \mathbb{R}_{+}$, Lipschitz on bounded subsets of $X$, and positive reals $p, \underline{c}$ and $\bar{c}$ such that

$$
\underline{c}\|x\|^{p} \leq V(x) \leq \bar{c}\|x\|^{p}, \quad \forall x \in X,
$$

and

$$
\bar{D}_{q} V(x) \leq-\|x\|^{p}, \quad \forall x \in X, \forall q \in \mathcal{Q} .
$$

Proof: We prove first the sufficiency part. Assume the existence of a continuous functional $V: X \rightarrow \mathbb{R}_{+}$ satisfying inequalities (24) and (25), with positive reals $p, \underline{c}$ and $\bar{c}$. Then, for all $x \in X, \sigma \in \mathcal{S}$ and $t \geq 0$, using inequality (25) we obtain (see [4, Theorem 9])

$$
V\left(T_{\sigma(\cdot)}(t) x\right) \leq V(x)-\int_{0}^{t}\left\|T_{\sigma(\cdot)}(\tau) x\right\|^{p} d \tau,
$$

from which we obtain, using inequality (24), the following

$$
\left\|T_{\sigma(\cdot)}(t) x\right\|^{p} \leq \frac{\bar{c}}{\underline{c}}\|x\|^{p}, \forall t \geq 0, \forall x \in X, \forall \sigma \in \mathcal{S} .
$$

Thus, the exponential boundedness property given by equation (7) is satisfied with $g(t) \equiv(\bar{c} / \underline{c})^{1 / p}$. By consequence, from the sufficiency part of Theorem 7 , it follows that

$$
\left\|T_{\sigma(\cdot)}(t) x\right\| \leq M e^{-\lambda t}\|x\|, \forall t \geq 0, \forall x \in X, \forall \sigma \in \mathcal{S},
$$

for some positive $M, \lambda$.

Let us prove now the necessity part. Assume that the system $\Sigma$ is 0 -UGES and let $M>0, \lambda>0$ be such that the inequality (6) holds. Choose $\gamma>0$ such that $2 \gamma-\lambda<0$. Without loss of generality, we can suppose that $M$ is sufficiently large in such a way that we have $\gamma>1 / 2 M^{2}$. Let $V: X \rightarrow \mathbb{R}_{+}$be the functional defined by

$$
V^{\gamma}(x)=\sup _{\sigma \in \mathcal{S}} \sup _{t \geq 0}\left\|e^{\gamma t} T_{\sigma(\cdot)}(t) x\right\|^{2}, \quad x \in X .
$$

Thanks to the uniform exponential stability of $\Sigma$, the functional $V$ is well defined. Let us check inequalities (24) and (25). The right-hand inequality in (24) follows directly from the 0-UGES assumption with $\bar{c}=M^{2}$. The left-hand inequality in (24) holds, with $\underline{c}=1$. Concerning inequality (25), observe that for all $h \geq 0, x \in X$ and for any $q \in \mathcal{Q}$ we have

$$
\begin{aligned}
& V^{\gamma}\left(T_{q}(h) x\right)=\sup _{\sigma \in \mathcal{S}} \sup _{t \geq 0}\left\|e^{\gamma t} T_{\sigma(\cdot)}(t) T_{q}(h) x\right\|^{2} \\
& =e^{-2 \gamma h} \sup _{\sigma \in \mathcal{S}} \sup _{t \geq 0}\left\|e^{\gamma(t+h)} T_{\sigma(\cdot)}(t) T_{q}(h) x\right\|^{2} \\
& \leq e^{-2 \gamma h} \sup _{\sigma \in \mathcal{S}} \sup _{t \geq 0}\left\|e^{\gamma(t+h)} T_{\sigma(\cdot)}(t+h) x\right\|^{2} \\
& \leq e^{-2 \gamma h} \sup _{\sigma \in \mathcal{S}} \sup _{t+h \geq 0}\left\|e^{\gamma(t+h)} T_{\sigma(\cdot)}(t+h) x\right\|^{2} \\
& =e^{-2 \gamma h} V^{\gamma}(x) .
\end{aligned}
$$

Therefore, for all $x \in X$ and any $q \in \mathcal{Q}$, it follows that

$$
\begin{aligned}
& \bar{D}_{q} V^{\gamma}(x)=\limsup _{h \rightarrow 0^{+}} \frac{V^{\gamma}\left(T_{q}(h) x\right)-V^{\gamma}(x)}{h} \\
& \leq \limsup _{h \rightarrow 0^{+}} \frac{e^{-2 \gamma h}-1}{h} V^{\gamma}(x) \\
& \leq \limsup _{h \rightarrow 0^{+}} \frac{e^{-2 \gamma h}-1}{h} M^{2}\|x\|^{2} \\
& =-2 \gamma M^{2}\|x\|^{2} \leq-\|x\|^{2},
\end{aligned}
$$


which implies that inequality (25) holds, with $p=2$.

Let us prove that the functional $V^{\gamma}$ is Lipschitz on bounded subsets of $X$. For this, let $r$ be any positive real and let $x, y \in B_{X}(0, r)$. We have

$$
\begin{aligned}
& \left|V^{\gamma}(x)-V^{\gamma}(y)\right|= \\
& \left|\sup _{\sigma \in \mathcal{S}} \sup _{t \geq 0}\left\|e^{\gamma t} T_{\sigma(\cdot)}(t) x\right\|^{2}-\sup _{\sigma \in \mathcal{S}} \sup _{t \geq 0}\left\|e^{\gamma t} T_{\sigma(\cdot)}(t) y\right\|^{2}\right| \\
& \leq \sup _{\sigma \in \mathcal{S}} \sup _{t \geq 0}\left|\left\|e^{\gamma t} T_{\sigma(\cdot)}(t) x\right\|^{2}-\left\|e^{\gamma t} T_{\sigma(\cdot)}(t) y\right\|^{2}\right|,
\end{aligned}
$$

from which we obtain, using the fact that $2 \gamma-\lambda<0$, the following inequalities

$$
\begin{aligned}
& \left|V^{\gamma}(x)-V^{\gamma}(y)\right| \\
& \leq 2 M r \sup _{\sigma \in \mathcal{S}} \sup _{t \geq 0}\left\|T_{\sigma(\cdot)}(t) x-T_{\sigma(\cdot)}(t) y\right\| \\
& \leq 2 M r L_{r}\|x-y\| .
\end{aligned}
$$

This implies that the functional $V^{\gamma}$ is Lipschitz on bounded subsets of $X$.

Remark 9: The construction of the Lyapunov functional (27), given in the proof of Theorem 8, is based on the classical construction given in [14] in the context of linear $C_{0}$-semigroups. This is also used in [10] in order to construct a coercive ISS Lyapunov functional for bilinear infinite-dimensional systems with bounded input operators. An alternative construction of a coercive common Lyapunov functional can be given, starting from the non-coercive Lyapunov functional given by (19), by the following

$V(x)=\sup _{\sigma \in \mathcal{S}} \int_{0}^{\infty}\left\|T_{\sigma(\cdot)}(t) x\right\|^{2} d t+\sup _{\sigma \in \mathcal{S}} \sup _{t \geq 0}\left\|T_{\sigma(\cdot)}(t) x\right\|^{2}$,

which guarantees that $V$ is the square of a norm; in this case the right-hand inequality in (24) follows directly from the 0-UGES assumption with $\bar{c}=M^{2}(1+1 / 2 \lambda)$, and the left-hand inequality in (24) holds, with $\underline{c}=1$, for $t=0$.

\section{CONCLUSION}

In this paper, we establish two converse Lyapunov theorems regarding global uniform exponential stability of nonlinear infinite-dimensional switching systems. In the first result we prove the existence of a noncoercive Lyapunov functional while the second result uses a coercive Lyapunov functional. A common tool for the arguments of two theorems is a generalization of the well-known Datko lemma to the case of nonlinear infinite-dimensional switching systems. Further work in the context of nonlinear infinite-dimensional switching systems will address issues such as stability of interconnected systems and converse Lyapunov theorems for semi-global uniform exponential stability and for inputto-state stability.

\section{REFERENCES}

[1] Michael S. Branicky. Multiple Lyapunov functions and other analysis tools for switched and hybrid systems. IEEE Transactions on Automatic Control, 43(4):475-482, 1998.

[2] Richard Datko. Extending a theorem of A. M. Liapunov to Hilbert space. Journal of Mathematical Analysis and Applications, 32:610-616, 1970.

[3] Wijesuriya P. Dayawansa and Clyde F. Martin. A converse Lyapunov theorem for a class of dynamical systems which undergo switching. IEEE Transactions on Automatic Control, 44(4):751-760, 1999.

[4] John W. Hagood and Brian S. Thomson. Recovering a function from a Dini derivative. American Mathematical Monthly, 113(1):34-46, 2006.

[5] Ihab Haidar, Paolo Mason, and Mario Sigalotti. Converse Lyapunov-Krasovskii theorems for uncertain retarded differential equations. Automatica, 62:263-273, 2015.

[6] Ihab Haidar, Paolo Mason, and Mario Sigalotti. Stability of interconnected uncertain delay systems: a converse Lyapunov approach. In G. Valmorbida, A. Seuret, I. Boussaada, and R. Sipahi, editors, Delays and Interconnections: Methodology, Algorithms and Applications, volume 10 of Advances in Delays and Dynamics. Springer, to appear.

[7] Falk M. Hante and Mario Sigalotti. Converse Lyapunov theorems for switched systems in Banach and Hilbert spaces. SIAM J. Control Optim., 49(2):752-770, 2011.

[8] Akira Ichikawa. Equivalence of Lp stability and exponential stability for a class of nonlinear semigroups. Nonlinear Analysis, Theory, Methods and Applications, 8(7):805-815, 1984.

[9] Birgit Jacob, Andrii Mironchenko, Jonathan R. Partington, and Fabian Wirth. Remarks on input-to-state stability and noncoercive Lyapunov functions. In 2018 IEEE Conference on Decision and Control (CDC), pages 4803-4808, Dec 2018.

[10] Andrii Mironchenko and Fabian Wirth. A note on input-to-state stability of linear and bilinear infinite-dimensional systems. In 2015 54th IEEE Conference on Decision and Control (CDC), pages 495-500, 2015.

[11] Andrii Mironchenko and Fabian Wirth. Existence of noncoercive Lyapunov functions is equivalent to integral uniform global asymptotic stability. Mathematics of Control, Signals, and Systems, 31(2):1-26, 2019.

[12] Andrii Mironchenko and Fabian Wirth. Non-coercive Lyapunov functions for infinite-dimensional systems. Journal of Differential Equations, 266(11):7038-7072, 2019.

[13] Amnon Pazy. On the applicability of Lyapunov's theorem in Hilbert space. SIAM Journal on Mathematical Analysis, 3(2):291-294, 1972.

[14] Amnon Pazy. Semigroups of Linear Operators and Applications to Partial Differential Equations. Springer-Verlag, New York, 1983.

[15] Eduardo D. Sontag and Yuan Wang. On characterizations of the input-to-state stability property. Systems \& Control Letters, 24(5):351-359, 1995.

[16] Roberto Triggiani. A sharp result on the exponential operatornorm decay of a family of strongly continuous semigroups. Semigroup Forum, 49(1):387-395, Dec 1994. 